\title{
An Efficient Representation of Enriched Temporal Trajectories
}

\author{
Nieves R. Brisaboa $\circledast^{\mathrm{a}}$, Antonio Fariña $\circledast^{\mathrm{b}}$, Diego Otero-González $\bigoplus^{\mathrm{c}}$ and Tirso V. Rodeiro $\bigodot^{\mathrm{d}}$ \\ Universidade da Coruña, CITIC, Fac. Informática, Database Lab. Elviña, 15071, A Coruña, Spain
}

Keywords: Compression, Data Management, Trajectories, Correlated Sequences.

\begin{abstract}
We present a novel representation of enriched trajectories of a mobile workforce management system. In this system, employees are tracked during their working day and both their routes and the tasks performed at each time instant are recorded. Our proposal tackles the representation of this information paying special attention to the space footprint without neglecting query time. We performed experiments using real and synthetic datasets where we show the compression effectiveness as well as the efficiency at query time. Our results showed that our proposal yields promising results in terms of the space needed to represent both users' locations and activities while performing access queries to the original data within microseconds.
\end{abstract}

\section{INTRODUCTION}

In this modern society, we all rely on devices in order to take part in this entangled dough the globalized world has become. It has been reported that more than $65 \%$ of the adults in advanced economies are owners of a smartphone but it also stands out that most devoted social network users are found in regions with lower internet rates (Poushter, 2019).

Thus, we are constantly producing a large amount of data that can be exploited in order to optimize a wide range of tasks and ease our daily life. Accordingly, an interest for programs able to handle trajectories and geographical information systems has grown in recent years giving birth to all kind of algorithms and systems that are able to locate mobile objects in real time or recover any past trajectory attending some user-defined criteria.

The need for studying trajectories not only as a sequence of time-stamped GPS points but also with a higher abstraction had led to the appearance of the socalled semantic trajectories in several works (Mountain and Raper, 2001; Yan et al., 2013; Parent et al., 2013). Fundamentally, this modern concept defines a split trajectory where each segment can be classified into different groups according to a defined parameter (location, activity, altitude, etc.). After the segmentation, a semantic label ("visiting a museum",

\footnotetext{
a (iD) https://orcid.org/0000-0001-8025-3048

b (iD https://orcid.org/0000-0001-8263-3298

c (iD) https://orcid.org/0000-0001-6631-4975

d(D) https://orcid.org/0000-0003-2373-0746
}

"having lunch at a service area", "driving through a traffic jam", etc.) is assigned to each trajectory segment (dos Santos Mello et al., 2019). Therefore, semantic trajectories are complex objects composed by the spatio-temporal polylines of each trajectory and textual tags identifying the aspect of interest of each segment.

Even though this approach enables abstract analysis and eases the organization of space-time information, there are no standard representations yet. Therefore, it is an essential need to find a way to store this vast amount of data (and metadata) following a compact approach to reduce the space footprint without disregarding the efficient query performance.

Commercial Geographical Information Systems (GIS) solve this problem by storing all the enriched segments of a trajectory in a traditional database containing the actual geometry besides all the defining attributes (semantic tag, initial timestamp, etc.). Despite using some geographical optimizations, GIS solutions can become rather inefficient due to the large size of the involved tables and its associated cumbersome handling (Alvares et al., 2007).

In this paper, we propose a novel technique to store and exploit enriched trajectories to support daily monitoring in a mobile workforce management scenario. Despite the fact that our proposal is a generalpurpose system that would fit in several domains (e.g. tourism activities, urban mobility data of vehicles, etc.), we have defined it as part of a bigger system that aims at optimizing the daily operation of the workers of an organic-waste management company. One 
of our main requirements was to supervise employee tasks in order to gather all possible information to detect any possible malfunction (e.g. if some employees regularly leave the planned route due to slow traffic it could be helpful to update the planner system). Therefore, our work aims to introduce a new structure able to track the trajectories of employees and the actual task they were performing at each time instant. As other works suggest, particularly when movements are not done in free space, there is no need to store explicit spatial geometries but only position identifiers which can be easily geolocated: e.g. segments from a road network (Brisaboa et al., 2020), rooms inside a building (Wyffels et al., 2014), etc.

Using this approach, the baseline for our structure are two correlated sequences that represent the location, and the activity done at each time instant by each user; i.e. one is an ordered list of the road segments traversed, and the other one is the sequence of activities performed at each segment (working at a client place, slow transit on planned route, taking a break, etc.). Thus, our proposal introduces a unique structure that represents temporal, spatial, and semantic knowledge and supports solving queries such as "indicate which tasks were performed by driver $X$, and which route did this driver follow to accomplish them yesterday from 14:30 to 16:00" Also, our system may solve simpler queries involving a combination of the associated dimensions; e.g. "where was driver K last Tuesday at 09:20?" or "what trajectory did driver T follow this morning?”

\section{BACKGROUND}

The problem of storing semantic trajectories efficiently has been of interest for more than one decade. This idea was already tackled in (Schmid et al., 2009) where a simplified trajectory representation was presented. In that proposal, they only stored the relevant points of the path through the network along with inferred timestamps instead of the original trajectory points.

More recently, a framework that divided trajectories into two separate representations considering their spatial and temporal components was introduced (Song et al., 2014). They also proposed a two-stage error-free spatial compression algorithm and an errorbounded temporal compression algorithm.

A compressed representation for semantic trajectories was presented in (Gao et al., 2019), where they used clustering to create regions of interest attending to those neighboring points that share the same semantic values, and then proposed a hierarchical multi- resolution network that enables compressing the semantic trajectories as a sequence of semantic regions of interest.

Meanwhile, the raise of repetitive datasets has led to the increasing development of compression techniques targeting the exploitation of such repetitiveness as a way to reduce their size. Despite the importance of reducing space, usually this it not the only concern as compressing data (to reduce their space needs) is rather useless if a decompression step is required before querying, as this would slow down any further exploitation of such data. This spatiotemporal trade-off is one of the main targets of a recent research field focused on the development of compact data structures.

Compact data structures (Navarro, 2016) are a family of general-purpose data structures that merge a compressed data representation with efficient query support. The main advantage of these structures relies on achieving competitive query times while using almost as little space as in its purely compressed representation.

Compact data structures have enhanced all kind of solutions, from document data mining (Fujishige et al., 2019) to bioinformatics (Crawford et al., 2018). In the scope of this work, there are some previous works tackling trajectory representations through compact data structures. Among them, an efficient representation for trajectories in free space was proposed in (Brisaboa et al., 2019). Using a system based on snapshots that store the positions of the objects at some (regular) given time instants and additionally keeps their relative movements between two consecutive snapshots, they are able to solve a range of spatiotemporal queries based on the movement of objects in a grid. A multidimensional representation for handling event sequences has also been proposed in the area of semantic trajectories (Brisaboa et al., 2018). That work presents a data reorganization strategy that aims at grouping/ordering the information of interest attending one (or several) of its semantic tags, and leads to an efficient representation to solve aggregation queries.

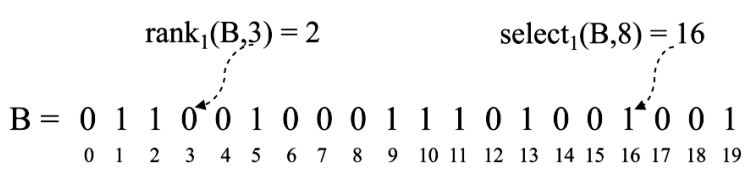

Figure 1: A bitvector $B$ and its two operations: rank and select.

One common basic component of these structures are bitvectors (or bit sequences). A bitvector $B[1, n]$ is a sequence of zeros and ones of length $n$, where the following two basic operations (also depicted in 
Figure 1) are expected to be supported:

- $\operatorname{rank}_{1}(B, i)$ returns the number of bits set to 1 in $B[1 . . i]$. Alternatively, $\operatorname{rank}_{0}(B, i)=i-$ $\operatorname{rank}_{1}(B, i) . \quad$ Note that $B[i]=\operatorname{rank}_{1}(B, i)-$ $\operatorname{rank}_{1}(B, i-1)$.

- $\operatorname{select}_{1}(B, i)$ returns the position in $1 . . n$ where the $i$-th 1 occurs. Therefore, $\operatorname{rank}_{1}\left(B, \operatorname{select}_{1}(B, i)\right)=$ $i$.

Similarly, operations $\operatorname{rank}_{0}(B, i)$ and $\operatorname{select}_{0}(B, i)$ can be defined to respectively count the number of zeros up to a given position, or locating the position in $B$ of the $i$-th zero.

Compressed bitvector representations exist (Raman et al., 2002; Okanohara and Sadakane, 2007; Navarro, 2016). However, in this work we have used plain bitvectors with select and rank support, achieving both operations a time complexity of $O(1)$ by using just $o(n)$ additional bits of space (Munro, 1996). Besides, we have used a special operation known as selectNext to $_{1}$ speed up consecutive select $_{1}$ queries (Navarro, 2016). Basically, if $B[i]$ contains a one, $j=\operatorname{selectNext}(B, i)$ returns the position $i(i>i)$ of the next one in $B$.

Despite the good computation prope tors offer, compact data structures rely or pression methods to effectively store the information. One of the most popular c algorithms used in the context of repetitiv Re-Pair (Larsson and Moffat, 1999). Th exploits pair repetition on a sequence rely tionary of rules. Basically, each pair of curring at least twice is substituted by a r hence shortening that sequence. In additic lating both the pair and the new symbol allow further decompression. Re-Pair als ceeds as follows:

- The most frequent pair $x y$ in a given is located.

- A new rule $X \rightarrow x y$ is created and stored in a dictionary of rules $D$.

- Each occurrence of the original pair is replaced in $T$ by $X$.

- The previous three steps are repeated recursively until there are no repeated pairs in $T$.

As an example to illustrate the behaviour of RePair, let us consider the sequence $T=\langle$ onabonana $\rangle$ and $D=\emptyset$, we can see that the most common pair of symbols in $T$ is $n a$. Thus, the algorithm generates a new rule $R_{1}: A \rightarrow n a$, which is added to the dictionary $D$, and replaces all the occurrences of $n a$ in $T$ by $A$. The resulting sequence now becomes

$T^{\prime}=\langle o A b o A A\rangle$, and $D=\left\{R_{1}: A \rightarrow n a\right\}$. This process is repeated recursively until the text contains no more repeated pairs. In our example, the next generated rule is $R_{2}: B \rightarrow o A$, transforming the text into $T^{\prime \prime}=\langle B b B A\rangle$, and $D=\left\{R_{1}: A \rightarrow n a ; R_{2}: B \rightarrow o A\right\}$. The algorithm ends due to the lack of repeated pairs.

\section{OUR PROPOSAL}

As stated before, the main idea of this work arises as part of a real mobile workforce management system in the scope of a organic-waste handling company where not only the trajectories of their employees are of interest but also the activity performed by them at each spatio-temporal point. Since the range of possible daily tasks performed by waste management truck drivers is very restricted, we were able to reduce the list to only 8 activities: being at headquarters, visiting a customer place, taking a break, normal/slow driving on un/planned route, and inactive. Thus, our problem can also be seen as the

$\mathrm{R}:$\begin{tabular}{|l|l|l|l|l|l|l|l|l|l|l|l|l|l|l|l|l|}
\hline $\mathrm{A}$ & $\mathrm{A}$ & $\mathrm{A}$ & $\mathrm{A}$ & $\mathrm{A}$ & $\mathrm{A}$ & $\mathrm{A}$ & $\mathrm{C}$ & $\mathrm{C}$ & $\mathrm{C}$ & $\mathrm{C}$ & $\mathrm{C}$ & $\mathrm{C}$ & $\mathrm{C}$ & $\mathrm{C}$ & $\mathrm{C}$ & $\mathrm{D}$ \\
\hline $\mathrm{A}: \mathrm{X}$ & $\mathrm{X}$ & $\mathrm{X}$ & $\mathrm{X}$ & $\mathrm{Y}$ & $\mathrm{Y}$ & $\mathrm{Y}$ & $\mathrm{Z}$ & $\mathrm{Z}$ & $\mathrm{Y}$ & $\mathrm{Y}$ & $\mathrm{Y}$ & $\mathrm{Y}$ & $\mathrm{Y}$ & $\mathrm{Z}$ & $\mathrm{Z}$ & $\mathrm{Z}$ \\
\hline
\end{tabular}

Figure 2: Original representation of both correlated sequences: road segments traversed $(R)$ and activities performed $(A)$.

A straightforward (naive) approach of our proposal would be to handle two aligned vectors containing the value of each dimension during a discretized time period, as depicted in Figure 2. Therefore, we would have a vector $R$ saving the identifiers of the road segments traversed by an employee and a second vector $A$ containing the activities performed by the worker. As the company tracks both values at every minute thanks to the driver's mobile phone, each element in both sequences represents a time interval of 60 seconds.

This naive solution contains a high degree of redundancy that can be exploited for the sake of compression. Given that each symbol in the previous sequences is represented with an integer identifier, it is a priority to reduce the number of repeated symbols of a sequence. This can be achieved with what we call a header vector, that is, a vector which avoids consecutive repeated symbols as $R_{H}$ in Figure 3. Using only this header vector it would be possible to retrieve 
the route followed by a worker on the actual chronologically ordered sequence of activities an employee has completed in a day. However, recall that the naive representation keeps the ordered sequence of events and, through consecutive repetitions, how much time was spent in each location/activity. This information would not be kept by storing just $R_{H}$ (or $A_{H}$ for activities) as indicated in Figure 3 . In order to retain this feature the header vector needs to be complemented with a bitvector $B$ representing each time interval as in

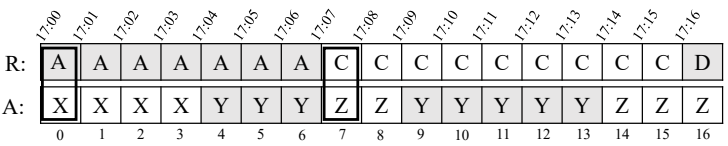

$$
\begin{aligned}
& \mathrm{R}_{\mathrm{H}}: \begin{array}{|c|c|c|}
\hline \mathrm{A} & \mathrm{C} & \mathrm{D} \\
\hline 0 & 1 & 2
\end{array} \\
& \text { B: } \begin{array}{|l|c|c|c|c|c|c|c|c|c|c|c|c|c|c|c|c|}
\hline 1 & 0 & 0 & 0 & 0 & 0 & 0 & 1 & 0 & 0 & 0 & 0 & 0 & 0 & 0 & 0 & 1 \\
\hline 0 & 1 & 2 & 3 & 4 & 5 & 6 & 7 & 8 & 9 & 10 & 11 & 12 & 13 & 14 & 15 & 16
\end{array}
\end{aligned}
$$

Figure 3: Example illustrating how we can decrease the space needs of $R$, by using $R_{H}$ and a bitvector $B$.

It is important to note that we work with two correlated sequences $R$ and $A$ in our particular context, so we need to store information about both sequences. Keeping in mind the idea of reducing space, instead of using two header vectors and two bitvectors, we can store two header vectors $R_{H}$ and $A_{H}$ and only one smaller structure to determine symbol repetitions. The main problem we have to deal with by following this approach, is that we have to be able to quickly identify changes in each sequence and, particularly, changes that occur simultaneously (in both sequences at the same time). To tackle that, we use a bitvector identifying a transition (in activities, trajectories or both) and, at least, another bitvector to discriminate which sequence has changed. In order to achieve this purpose, we introduce three different bitvector-based solutions:

- 3 Bitvectors (3B): This approach uses three bitvectors to detect changes in both sequences. The first bitvector $(C)$ identifies change events in any vector. The second bitvector $(D)$ discriminates between single changes in vector $R$ (with greater change probability). Last bitvector $\left(D_{2}\right)$ differentiates between single changes in sequence $A$ and double changes (in both $R$ and $A$ ) at the same time instant.

- Occurrence Pointers (OP): This solution shares the same bitvector $C$ to identify changing time instants in both vectors $R$ and $A$, and uses $D$ to discern which sequence has changed. The events of double changes at the same instant are handled with a new array $P$ that keeps pointers to the orig-

\begin{tabular}{|c|c|c|c|c|c|c|c|c|c|c|c|c|c|c|c|c|c|}
\hline & $\mathrm{R}_{\mathrm{l}}$ & $: 2$ & & & D & & & & & $\mathrm{A}_{\mathrm{H}}$ & & & & & $y$ & & \\
\hline & & & & & & & & & bitt & ectors & & & & & & & \\
\hline & & & & & & & & & & & & & & & & & \\
\hline C: & 1 & 0 & 0 & 0 & 1 & 0 & 0 & 1 & 0 & 1 & 0 & 0 & 0 & 0 & 1 & & 1 \\
\hline D: & 1 & 0 & 1 & 0 & 0 & 1 & & & & & & & & & & & \\
\hline $\mathrm{D}_{2}$ & 1 & 1 & 0 & 0 & & & & & & & & & & & & & \\
\hline & & & & & & & & & ccur & rence & oin & & & & & & \\
\hline $\mathrm{C}:$ & 1 & 0 & 0 & 0 & 1 & 0 & 0 & 1 & 0 & 1 & 0 & 0 & 0 & 0 & 1 & & 1 \\
\hline D: & 1 & 0 & 1 & 0 & 0 & 1 & & & & & & & & & & & \\
\hline P: & 0 & 7 & & & & & & & & & & & & & & & \\
\hline & & & & & & & & & 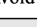 & . & & & & & & & \\
\hline C: & 1 & 1 & 0 & 0 & 1 & 0 & 0 & 1 & 1 & 1 & 0 & 0 & 0 & 0 & 1 & & 1 \\
\hline D: & 1 & 0 & 0 & 1 & 0 & 0 & 0 & 1 & & & & & & & & & \\
\hline
\end{tabular}
inal positions (within $C$ ) where they occurred.

Figure 4: Our proposal uses two common arrays $\left(R_{H}\right.$ and $A_{H}$ ) to store three dimensional information (including time), one bitvector $C$ to encode changes on them and one of the three types of auxiliary structures to manage twodimensional changes in $C$.

- Avoid Duplicity (AD): A simpler lossy method to tackle the concurrent changes problem would be to force them into different time instants. Thus, each double change event would be represented in $C$ as two consecutive changes, assigning in $D$ vector a one to each. It is important to notice that this approach may imply some inaccuracies that would translate into loss of detailed information due to the lack of a third level. In a context where this could become a usual issue, the fix could consists in using a finer granularity, i.e. to survey activities and locations every second instead of every minute so that double changes would become very unlikely.

\subsection{Handling Drivers' Work Shifts}

The above proposals permits us to handle the activities and locations of any given employee. However, our aim is to represent a complete dataset with the information corresponding to all the existing drivers along a large period of time of $K$ working days. Therefore, our bitvectors $C, D, D_{2}$, and vector $P$ could contain information corresponding to different drivers during up to $K$ distinct days, and we have to be able to distinguish which part of those structures belongs to each driver and day.

We use a little auxiliary structure in order to check the working days when each employee worked, as not 
all drivers work every day. In particular, we use a chronologically ordered bitvector $d x c$ containing $K$ bits for the first driver, other $K$ bits for the second driver, and so on, where a 1 indicates that an employee vxorlzed a given day and $\Omega$ maanc the onnocite

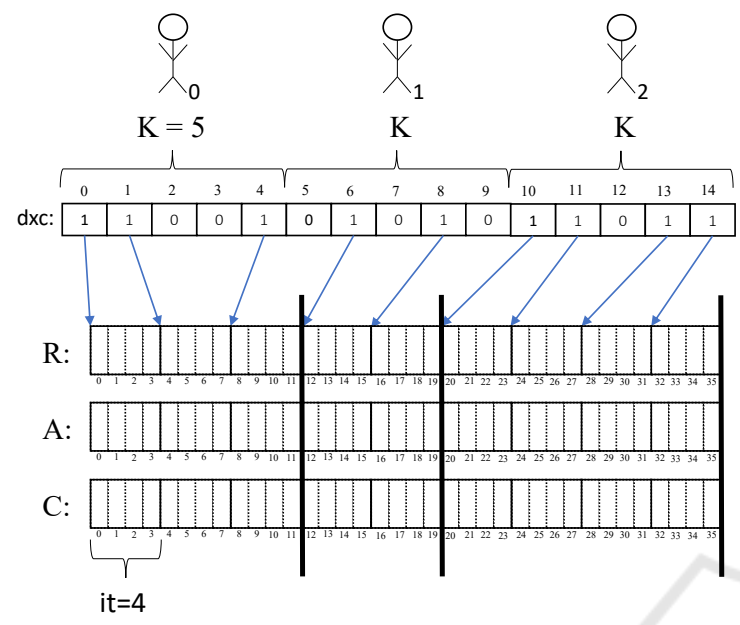

Figure 5: Bitvector $d x c$ identifies the days on which drivers have worked and enables easy acces to the corresponding offsets in road segments vector $(R)$, activity vector $(A)$ and changes vector $(C)$.

In practice, our auxiliary bitvector acts as an array of pointers to the initial position of a workday of a given driver in vectors $R, A$ and, $C$. In $d x c$, each driver has the same amount of workable days $K$, and each of those days is divided into a constant amount of discretized time intervals it. Therefore, it can be easily checked if a given driver worked on a given day by computing $w=d x c[($ driver $* K)+$ day -1$]$. If the employee worked that day $(w=1)$, the offset in $R$ (and also in $A$ and $C$ ) for this driver on that day can be obtained by accessing from position $l=i t * \operatorname{rank}_{1}(d x c,($ driver $* K)+$ day -1$)$ to position $r=l+i t-1$. We define an operation $[l, r] \leftarrow$ getRange (dxc, driver, day) accordingly.

As an example, let us consider Figure 5, where we store information for $K=5$ days and, for each day, we handle $i t=4$ time intervals. We can check if driver 1 worked on day 2 as $w=d x c[(1 * 5)+2-1]=$ $d x c[6]=1$. Once we have confirmed that the driver worked on that day, we can jump directly to the range defined by position $l=4 * \operatorname{rank}_{1}(d x c, 6)=12$ in $R$ (also for $A$ or $C$ ), and $r=12+4-1=15$. This range can be obtained directly as $[12,15] \leftarrow$ getRange $(d x c, 1,2)$, as defined above.

\subsection{Reducing Space Usage}

In the particular context of a mobile workforce management system, there will always be intrinsic global repetitiveness not considered in the previous section. Previously, we have defined some techniques to exploit symbol repetitions during a given workday of a single employee, i.e. we take advantage of long periods where a worker performed the same activity or traversed the same road/highway. However, if we analyze the behaviour of several employees globally, we may find again a high degree of repetitiveness between them. For example, examining the trajectories of every worker, it seems quite likely that they always start the route at headquarters and then traverse the same streets/roads (or at least a reduced set of them). The same applies to activities, it is expected that workers perform the same activity sequence, e.g. a driver leaves the planned route due to a traffic jam.

As detailed in Section 2, Re-Pair is a compression technique that exploits global repetitions. Expected that, by applying Re-Pair over our header vectors $R_{H}$ and $A_{H}$, we could be able to reduce their memory footprint even further, yet probably in exchange for a slower access time. Note that if we have $R_{H}$ and $A_{H}$ compressed with Re-Pair, each time we wanted to access any of their elements, we would have to decompress them from the Re-Pair compressed sequence.

\section{QUERIES}

We have selected four representative queries to show how our structure (considering its three variants 3B, $\mathrm{OP}$, and $\mathrm{AD}$ ) works. On the one hand, we offer access capabilities for routes and activities (RouteAtTime and ActivityAtTime); i.e. recovering the corresponding location/activity of a particular driver at a given time instant. On the other hand, we provide temporal window queries for both dimensions (RoutesAtTimeRange and ActivitiesAtTimeRange).

- RouteAtTime (RT): This query recovers the road segment identifier where a driver was in a specific instant; i.e. it permits us to obtain the location of any worker at a given time instant.

It takes three input parameters: driver, day, and time_instant. Thanks to the already explained calculations on the bitvector $d x c$ (see getRange operation in Section 3.1) we can obtain the positions $[1, \mathrm{r}]$ within bitvector $C$ that correspond to the information regarding the given day of a particular driver. Recall that range of positions is obtained as $[l, r] \leftarrow$ getRange $(d x c$, driver, day $)$. 
Then, using the parameter time_instant we can get the position $I=l+$ time_instant of the queried time instant within the day. The number $(\mathrm{S})$ of ones in bitvector $C$ until the position $I$ (computed as $S=\operatorname{rank}_{1}(C, I)$ ) represents the total number of time instants when there were changes (routes and activities) until the time instant of interest. That is, changes within $R[0, I]$ or $A[0, I]$.

As explained before, bitvector $D$ allows to discriminate routes information. Thus, we can easily obtain the total amount of road segment changes until the queried instant as $J=\operatorname{rank}_{1}(D, S)$. Therefore, $J-1$ represents the position on $R_{H}$ where the road segment identifier is stored.

- RoutesAtTimeRange (RTR): This query reconstructs the trajectory of a driver for a specific interval of time. With this query we are able to obtain the driver's route in a given working day. It takes three input parameters: driver, day, and an interval_time $[X . . Y]$.

We can use the previous query to find the first road segment identifier of the queried interval $[X . . Y]$ by just computing RouteAtTime(driver, day, $X$ ). During that process, recall we obtain the corresponding position $I$ within $C$, and $S=\operatorname{rank}_{1}(C, I)$ within $D$ corresponding to the last road change that occurred either before or at time instant $X$. However, in order to retrieve the full trajectory of a driver during the given interval, we need to traverse the ones within $D$ from $S$ on using selectNext $1(D, S)$; i.e. jumping to the following road segment changes marked on $D$. Note that this process must end once we have gone further than the corresponding position $E=I+(Y-X)$ associated to $Y$ in $C$.

Therefore, we start with the same $I$ and $S=\operatorname{rank}_{1}(C, I)$ values obtained by RouteAtTime(driver,day, $X$ ), and also output the (initial) road segment identifier within $R_{H}\left[\operatorname{rank}_{1}(D, S)-1\right] n$ times, where $n=$ select $\operatorname{ext}_{1}(D, S)-S-1$. Then, while it holds that $I \leq E$, we set $n=$ selectNext $_{1}(D, S)-S-1$; $S=\operatorname{selectNext}_{1}(D, S)$ (hence looking for the next road segment change in $D$ ); we output the road segment identifier in $R_{H}\left[\operatorname{rank}_{1}(D, S)-1\right] n$ times; and finally compute $I=\operatorname{select}_{1}(C, S)$ to map the position $S$ from $D$ into $C$, which allows us to know if the process must either end $(I>E)$ or handle the next road-segment change otherwise.

- ActivityAtTime (AT): This query allows us to return the activity being performed by a driver at a specific instant. It takes three input parameters: driver, day, and time_instant.
This query is rather similar to RouteAtTime but instead of just using bitvectors $C$ and $D$, this time it will be necessary to reach the deepest bitvectors. Recall that Section 3 already introduced our three strategies to deal with double changes (road segment and activity changing at the same instant).

We can get the position in $D$ as we did in the previous queries (recall how $S$ and $I$ were initialized for RouteAt Time operation). However, $D$ does not discriminate between activity identifiers and double changes, and here is where our alternatives differ:

- 3 Bitvectors $(3 B)$ : Uses a third bitvector $D_{2}$ to be able to compute if the $i$-th one in $D$ corresponds only to a road segment change $\left(D_{2}[i]=\right.$ $0)$ or to a double change $\left(D_{2}[i]=1\right)$. It is important to note that, for this query, this alternative needs to consider single activity changes (zeros in $D$ ) and double changes (ones in $D_{2}$ ) up to position $S$ (where $S$ was the position in $C$ associated to the time instant of interest). Therefore, the amount of double changes can be computed as $D C=\operatorname{rank}_{1}\left(D_{2}, \operatorname{rank}_{1}(D, S)\right)$, while single activity changes can be counted as $A C=S-\operatorname{rank}_{1}(D, S)$. Finally, $D C+A C$ gives us the total amount of activity changes until the queried time instant. Thus, we obtain the activity being performed by the given driver at the indicated time instant by accessing the value $A_{H}[D C+A C-1]$.

- Occurrence Pointers $(O P)$ : In this solution we use a list of pointers $P$ pointing at the position of ones in $C$ which correspond to a double change.

To obtain the total number of simultaneous changes $D C$ up to the position $I$ from $C$, we count all the values of $P$ that are below $I$ (using a binary search over $P$ that takes $O(\log |P|)$ time, to locate the last index $p$ such that $P[p] \leq$ $I)$. The single activity changes can be obtained as $A C=\operatorname{rank}_{0}(D, S)$. Concluding again that the driver was performing activity $A_{H}[D C+A C-$ 1].

- Avoid Duplicity (AD): This solutions unfolds simultaneous changes in two consecutive single changes, significantly simplifying the operations. Hence, the total amount of activity changes until the queried time instant can be obtained by simply computing $A C=\operatorname{rank}_{0}(D, S)$ and the searched activity is $A_{H}[A C-1]$. 
- ActivitiesAtTimeRange (ATR): This query allows us to return the sequence of activities of a given driver during a particular time interval. It also takes three input parameters: driver, day, and an interval_time $[X . . Y]$.

This query is rather similar to RoutesAtTimeRange, yet returning a list of activities instead of road segments. Therefore, as is the case with ActivityAtTime, it will be necessary to traverse the deepest bitvectors.

Again, we can calculate the initial position $S$ in $D$ discriminating the activities from the double changes as it has been explained before for operation ActivityAtTime. Then, each alternative representation will retrieve the list of activities in a different way:

- 3 Bitvectors (3B): As usual, the first activity identifier to return can be computed with ActivityInTime (driver, day, $X$ ), along with $I$ and $S$ positions that are obtained as in the previous explanations. Again, this alternative needs to check single activity changes (zeros in $D$ ) and double changes (ones in $D_{2}$ ). Therefore, the position of the next single activity change after $S$, may be calculated as $A C=\operatorname{selectNext}_{0}(D, S)$, while the next double change would be at position $D C=$ $\operatorname{select}_{1}\left(D, \operatorname{selectNext}_{1}\left(D_{2}, \operatorname{rank}_{1}(D, S)\right)\right)$.

Thus, the first activity of interest was performed during the interval $C\left[I, \operatorname{select}_{1}(C, \min (D C, A C))-1\right]$; i.e. it must be output $n=\operatorname{select}_{1}(C, \min (D C, A C))-I-1$ times. Then, the next activity in $A_{H}$ starts, so we update $I=\operatorname{select}_{1}(C, \min (D C, A C))$, compute the new $A C$ and $D C$ positions to obtain again the smallest value, and the process would be repeated until we have gone further than the corresponding position $E=I+(Y-X)$ associated to $Y$ in $C$ (as in RoutesAtTimeRange).

- Occurrence Pointers (OP): To get the value of the first activity change in the time interval $[\mathrm{X} . . \mathrm{Y}]$ we use the query ActivityInTime (driver, day, $X$ ). Then, $A C$ can be computed as in the previous alternatives (using selectNext $t_{0}$ in $D$ ) while $D C$ would be the amount of all the pointers $P[i]$ that fall within the queried interval $\left[\operatorname{rank}_{1}(C, I), \operatorname{rank}_{1}(C, E)\right]$.

- Avoid Duplicity (AD): Due to the simplicity of this alternative, the calculations are practically the same as RoutesAtTimeRange but this time operating over activity changes (zeros); hence, traversing $D$ at positions $S, S^{\prime}=$ selectNext ${ }_{0}(D, S)$, and so on.

\section{EXPERIMENTAL EVALUATION}

This section describes the two datasets (one for route identifiers and another one for activity identifiers) that have been used to test our structure in terms of compression effectiveness and performance at query time. We analyze the results obtained, comparing the three variants of our proposal. Finally, Section 5.2.3 includes our experiments to show the results obtained when Re-Pair is applied on the header vectors $R_{H}$ and $A_{H}$.

\subsection{Datasets}

We can distinguish two types of datasets used along this work: one real dataset that consists of the positions of the employees of our collaborating company during their workday, and two synthetic activity datasets to test different scenarios.

The first one was obtained from the GPS data stored by the mobile phones of the truck drivers during their routine gathering waste and, essentially, it is a chronologically ordered list of road segment identifiers that are recorded at discretized time intervals of 60 seconds. The resulting number of entries in $R$ was $14,439,424$ and the number of different road segments handled was 101,623 .

The second type of datasets are synthetic datasets that we generated due to the lack of real activity data. Using the 8 activities described in Section 3, we designed these synthetic datasets resembling normal employees behavior as much as possible. To ensure the realism of this data, we developed a complex system where each road segment is assigned a subset of possible activities based on its geolocation (e.g. the activity "being at headquartes" cannot be possible outside the company facilities). Since we were generating the activities associated to each driver along each road segment present in $R$, the number of entries in $A$ was also $14,439,424$.

With the aim of testing a wide range of scenarios we created two activity datasets attending to different degrees of variability. Note that the behaviour of our proposal may differ depending on the amount of activity changes (both, compression ratios and access times). We created two activity datasets: one with a low rate of variability (there is a change in the value of the activity every 100 instants of time) and another dataset with a higher variability (there is a change in the value of the activity every 10 instants of time). 


\subsection{Experimental Results}

We have run experiments using the three variants of our proposal $(3 \mathrm{~B}, \mathrm{OP}$, and $\mathrm{AD})$ to compare their compression effectiveness and performance to access the original information. Our experiments were run on an isolated computer using an Intel Xeon ES2470@2.30GHz processor (20 MB of cache) and 64 GB of RAM. It runs Debian 10.8 (buster) with kernel 5.4.0 (64 bits). The compiler used was g++ version 7.4.0, with $\mathrm{C}++17$ option $(-\mathrm{std}=\mathrm{c}++17)$.

As a baseline for our comparison we have implemented the naive solution depicted in Figure 2. This simple approach uses just two symbol sequences (road segment identifiers and activity identifiers). To make this proposal more competitive in terms of space needs, we have chosen an efficient bitcompaction representation for these sequences, in which all identifiers are stored using the minimum number of bits $b$ needed to represent the maximum value of the vector $u$ (i.e. $b=\left\lceil\log _{2} u\right\rceil$ bits). Our bit-wise compacted vectors for $A$ and $R$ were implemented using the SDSL library (Gog et al., 2014). Recall that since the number of different road segments is 101,623 we used only $b=17$ bits per road segment identifier. Similarly, since we had only 8 different activities, 3 bits per activity identifier were used. Consequently, the overall size of the baseline representation of the sequence $R$ was $30.7 \mathrm{MiB}$, while for sequence $A$ we used $1.8 \mathrm{MiB}$, totalizing $32.5 \mathrm{MiB}$.

The same bit-wise compaction strategy was also applied to our proposal, where both header vectors, $R_{H}$ and $A_{H}$, were stored using bit-wise SDSL vectors.

\subsubsection{Compression Results}

As stated before, synthetic activity datasets were used to test our proposal under different scenarios with two different degrees of repetitiveness (higher or lower amount of consecutive repeated values).

Table 1: Compression ratios as $\%$ of the baseline representation using the activity dataset with a low repetitiveness (one change every 10 activities).

\begin{tabular}{|c|c|c|}
\hline $3 \mathrm{~B}$ & OP & AD \\
\hline 26.69 & 32.24 & 26.40 \\
\hline
\end{tabular}

Table 1 shows how the space needs ${ }^{1}$ of our three alternative representations using an activity dataset with low repetitiveness (activities change once every

\footnotetext{
${ }^{1}$ We show compression ratio as the percentage of the size of the compressed representation $(c)$ with respect to the baseline representation $(s)$ of the source data; i.e. $100 \times$ $c / s$.
}

10 activities). As we can see, the best compression results are obtained by the $A D$ solution (26.40\%), followed closely by $3 B(26.69 \%)$. On the other hand, the pointer vector of $O P$ negatively affects its space footprint (32.24\%).

Table 2 depicts the compression ratios for our proposals but this time using an activity dataset with high repetitiveness ratio (activities change once every 100 activities). $A D$ is still the most compact approach, but now results are much more similar because, with that low rate of activity changes, it is less likely a double shift to occur, i.e. third level vectors $\left(D_{2}\right.$ in $3 B$ and $P$ in $O P$ ) contain little data.

Table 2: Compression ratios as \% of the baseline representation using the activity dataset with a high repetitiveness (one change every 100 activities).

\begin{tabular}{|c|c|c|}
\hline 3B & OP & AD \\
\hline 11.71 & 11.71 & 11.48 \\
\hline
\end{tabular}

Comparing the results in Tables 1 and 2, we can see that the behaviour of our proposal is far better when applied to a repetitive scenario. This is mainly due to the fact that increasing the number of consecutive repeated values allows our proposal to reduce significantly the header vectors sizes. This also affects the bitvectors of our proposed variants since with a higher amount of consecutive repetitions the bitvectors $C$ and $D$ (see Figure 4) may become sparse bitvectors.

\subsubsection{Access Time Results}

With the aim of analyzing access time to our proposed structures we have tested the queries described in Section 4 . We compared the querying time obtained by the baseline representation with that of our three proposed alternatives. Again, all experiments were carried out using a real route dataset and two synthetic activity datasets.

Regarding route queries (RT and RTR), the first consideration we should note is that our three alternatives use roughly the same amount of time for both queries as all three solutions share the same underlying computation, that is, there is no need in any proposed alternative to reach the deepest vectors.

Table 3: Access times (ns) for route information.

\begin{tabular}{|c|r|r|r|r|}
\cline { 2 - 5 } \multicolumn{1}{c|}{} & Baseline & \multicolumn{1}{c|}{ 3B } & OP & AD \\
\hline RTR & 90.0 & 100 & 100 & 100 \\
\hline RT & 0.50 & 10 & 10 & 10 \\
\hline
\end{tabular}

Table 3 depicts how our solutions are not far from the baseline when accessing the road segments tra- 
versed by a driver during a (random) complete day that involves 600 minutes $(R T R)$. However, we are not so competitive when we perform an access $(R T)$ to retrieve the road segment identifier corresponding to a unique (random) time instant. In the latter, the baseline is capable of accessing any position of any vector in constant time, while our proposal needs to map the queried position in the header vector through the different bitvectors.

Concerning activities, query times corresponding to operations $A T$ and $A T R$ become worse because our proposal needs to roam deeper in our bitvector hierarchy. However, it is important to keep in mind that our structure may use up to $88 \%$ less space than the original representation. Tables 4 and 5 include the results obtained for our two synthetic activity datasets.

Table 4: Access times (ns) to the activity dataset with a low repetitiveness (one change every 10 activities).

\begin{tabular}{|c|r|r|r|r|}
\cline { 2 - 5 } \multicolumn{1}{c|}{} & Baseline & \multicolumn{1}{c|}{$3 \mathrm{~B}$} & OP & AD \\
\hline ATR & 90.00 & 200 & 210 & 180 \\
\hline AT & 0.70 & 98 & 165 & 90 \\
\hline
\end{tabular}

A lower degree of repetitiveness (i.e. having a large amount of activity changes) affects directly the amount of information stored in our proposal as header vectors increase proportionally and, consequently, the amount of ones stored in our bitvectors grows accordingly. This largely worsens ATR access times as the efficiency in our proposed bitvectors depend on the ability to jump between consecutive ones, skipping a huge amount of 0 's.

Table 5: Access times (ns) to the activity dataset with a high repetitiveness (one change every 100 activities).

\begin{tabular}{|c|r|r|r|r|}
\cline { 2 - 5 } \multicolumn{1}{c|}{} & Baseline & 3B & OP & AD \\
\hline ATR & 90.00 & 120 & 140 & 110 \\
\hline AT & 0.70 & 30 & 50 & 13 \\
\hline
\end{tabular}

Nonetheless, Table 5 shows that query times for $A T R$ become more competitive when a highly repetitive dataset is used, although we are still clearly slower than the baseline representation at $A T$ access queries.

Regarding the three different alternatives of our proposal, $A D$ is still the fastest variant as it has one less level to traverse during the query.

\subsubsection{Applying Re-Pair}

We applied Re-Pair over $R_{H}$ and $A_{H}$ sequences in our proposals as a way to exploit the expected underlying repetitiveness within them. We can see below that we obtained an improved compression ratio. How-
Table 6: Compression ratios (in \%) using Re-Pair on the activity dataset with a low repetitiveness ratio (one change every 10 activities).

\begin{tabular}{|c|c|c|}
\hline 3B & OP & AD \\
\hline 98.55 & 116.67 & 97.78 \\
\hline
\end{tabular}

ever, access times largely worsened due to the need of performing a Re-Pair decompression process from the beginning of the sequence to recover the original data.

In practice, to provide pseudo-random access to the compressed data we enriched our Re-Pair implementation with a list of pointers referencing the beginning of each day. This provided synchronization points at the beginning of each day and consequently sped up the partial decompression of our sequences $R_{H}$ and $A_{H}$ as a full decompression was no longer necessary.

Table 7: Compression ratios (in \%) using Re-Pair on the activity dataset with a high repetitiveness (one change every 100 activities).

\begin{tabular}{|c|c|c|}
\hline 3B & OP & AD \\
\hline 88.68 & 86.79 & 86.79 \\
\hline
\end{tabular}

Tables 6 and 7 present the compression ratios after applying Re-Pair in our solutions. For the sake of a fair comparison, all the results presented in this section have been measured against a compressed baseline using the same enriched Re-Pair algorithm. Note that, while the previous baseline required $30.7 \mathrm{MiB}$, our new baseline compressed with Re-Pair required 13.8 $\mathrm{MiB}$ in the lesser repetitive scenario and 5.3 MiB in the higher repetitive one. Therefore, Re-Pair was surprisingly successful in the non-repetitive scenario, where we could decrease the space requirements of the regular uncompressed baseline to around $44.9 \%$ of its size. In the most repetitive scenario, using Re-Pair led to even better results, reducing the space needs to around $17.2 \%$ of the original size.

Firstly, Table 6 details the Re-Pair compression results obtained for the lesser repetitive activity dataset. We can see that the results obtained by our proposals hardly improve the values of the baseline representation, and indeed, in the variant $O P$, space requirements are worsen than those of the baseline. Secondly, Table 7 describes a slightly better scenario regarding the high repetitiveness dataset as all our alternative representations overcome the results of the baseline.

The cost of reducing the space requirements is paid at query time because, as indicated above, partial decompression is required. Access time typically worsens by a constant factor $F$ of roughly around 
Table 8: Access times (ns) to the route dataset with Re-Pair.

\begin{tabular}{|c|r|c|c|c|}
\cline { 2 - 5 } \multicolumn{1}{c|}{} & Baseline & 3B & OP & AD \\
\hline RTR & 3090 & 3100 & 3100 & 3100 \\
\hline RT & 3001 & 3010 & 3010 & 3010 \\
\hline
\end{tabular}

3000 nanoseconds (see Tables 8, 9, and 10) corresponding to the decompression of either 600 road segments identifiers (RTR) or 600 activity values (ATR). Note that, for RT and AT, we are also paying that extra decompression cost because, in our current implementation, we are decompressing all the 600 values corresponding to a day, even though we are just retrieving one of those values. This is clearly and unoptimized operation that we will improve shortly by partially decompressing up to the queried time_instant of the day.

Table 9: Access times (ns) to the activity dataset with a low repetitiveness ratio (one change every 10 activities) with Re-Pair.

\begin{tabular}{|c|r|c|c|c|}
\cline { 2 - 5 } \multicolumn{1}{c|}{} & Baseline & 3B & OP & AD \\
\hline ATR & 3090 & 3200 & 3210 & 3180 \\
\hline AT & 3001 & 3110 & 3165 & 3090 \\
\hline
\end{tabular}

Again, apart from the extra decompression cost, the results obtained by our variants are similar to those in the previous sections where Re-Pair was not used.

Table 10: Access times (ns) to the activity dataset with a high repetitiveness (one change every 100 activities) with Re-Pair.

\begin{tabular}{|c|r|c|c|c|}
\cline { 2 - 5 } \multicolumn{1}{c|}{} & Baseline & 3B & OP & AD \\
\hline ATR & 3090 & 3120 & 3140 & 3110 \\
\hline AT & 3001 & 3040 & 3060 & 3025 \\
\hline
\end{tabular}

To sum up, we can see that, as expected, using RePair brings a space/time trade-off. We save space, yet our solution becomes clearly slower at query time.

\section{CONCLUSIONS AND FUTURE WORK}

We have presented a novel representation of enriched trajectories as part of a real mobile workforce management system. Our proposal includes three variants, where each of them employs a different bitvector-based solution in order to achieve a good trade-off between spatial footprint and query times.

Our proposal has been tested against real and synthetic datasets, using different distributions in the latter in order to study the behaviour of our proposal in diverse scenarios. Experiments have shown how our new representation is able to store large amounts of information using around a $88 \%$ less space than our baseline technique while obtaining competitive access times.

In addition, we have analyzed the use of a wellknown compression technique, known as Re-Pair, to boost the already good compression ratios achieved. Our experiments showed Re-Pair was able to reduce the size of our proposal even further, at the cost of slower access times.

As future work, our main aim would be to create another compression representation that could allow reduce even further the space requirements while retaining good performance at query time. Apart from that, we are also working on endowing our proposal with dynamic capabilities, i.e. enabling the information stored in it to grow over time.

\section{ACKNOWLEDGEMENTS}

Partially funded by the CITIC research center funded by Xunta/FEDER-UE 2014-2020 Program, grant ED431G 2019/01.

MICIU (PGE/ERDF) [Datos 4.0: TIN2016-78011C4-1-R; STEPS: RTC-2017-5908-7; BIZDEVOPS: RTI2018-098309-B-C32].

IGAPE/Xunta (FEDER-UE) 2014-2020 [IG240.2020.1.185].

Xunta/GAIN (ERDF) [GEMA: IN852A 2018/14] and by FPI Program [BES-2017-081390].

\section{REFERENCES}

Alvares, L. O., Bogorny, V., Kuijpers, B., de Macêdo, J. A. F., Moelans, B., and Vaisman, A. A. (2007). A model for enriching trajectories with semantic geographical information. In Proceedings of the ACM International Symposium on Geographic Information Systems, ACM-GIS 2007, page 22.

Brisaboa, N. R., de Bernardo, G., Navarro, G., Rodeiro, T. V., and Seco, D. (2018). Compact representations of event sequences. In Proceedings of the Data Compressioni Conference, DCC18, pages 237-246.

Brisaboa, N. R., Fariña, A., Navarro, G., and Rodeiro, T. V. (2020). Semantrix: A compressed semantic matrix. In Proceedings of the Data Compression Conference, DCC 2020, pages 113-122.

Brisaboa, N. R., Gómez-Brandón, A., Navarro, G., and Paramá, J. R. (2019). Gract: A grammar-based compressed index for trajectory data. Information Sciences, 483:106-135.

Crawford, V. G., Kuhnle, A., Boucher, C., Chikhi, R., and 
Gagie, T. (2018). Practical dynamic de bruijn graphs. Bioinformatics, 34(24):4189-4195.

dos Santos Mello, R., Bogorny, V., Alvares, L. O., Santana, L. H. Z., Ferrero, C. A., Frozza, A. A., Schreiner, G. A., and Renso, C. (2019). Master: A multiple aspect view on trajectories. Transactions in GIS, 23:805-822.

Fujishige, Y., Nakashima, Y., Inenaga, S., Bannai, H., and Takeda, M. (2019). An improved data structure for left-right maximal generic words problem. In Proceedings of International Symposium on Algorithms and Computation, ISAAC 2019, volume 149, pages 40:1-40:12.

Gao, C., Zhao, Y., Wu, R., Yang, Q., and Shao, J. (2019). Semantic trajectory compression via multi-resolution synchronization-based clustering. Knowledge-Based Systems, 174:177-193.

Gog, S., Beller, T., Moffat, A., and Petri, M. (2014). From theory to practice: Plug and play with succinct data structures. In Proceedings of International Symposium on Experimental Algorithms, SEA 2014, pages 326-337. Springer International Publishing.

Larsson, N. J. and Moffat, A. (1999). Offline dictionarybased compression. In Proceedings of the Data Compression Conference, DCC 1999, pages 296-305.

Mountain, D. and Raper, J. (2001). Modelling human spatio-temporal behaviour: a challenge for location based services. In Proceedings of the International Conference on GeoComputation, pages 65-74.

Munro, J. I. (1996). Tables. In Proceedings of the Conference on Foundations of Software Technology and Theoretical Computer Science, FSTTCS 1996, volume 1180 , pages $37-42$.

Navarro, G. (2016). Compact Data Structures: A Practical Approach. Cambridge University Press, USA, 1st edition.

Okanohara, D. and Sadakane, K. (2007). Practical entropycompressed rank/select dictionary. In Proceedings of the Meeting on Algorithm Engineering \& Expermiments (ALENEX 2007), pages 60-70.

Parent, C., Spaccapietra, S., Renso, C., Andrienko, G. L., Andrienko, N. V., Bogorny, V., Damiani, M. L., Gkoulalas-Divanis, A., de Macêdo, J. A. F., Pelekis, N., Theodoridis, Y., and Yan, Z. (2013). Semantic trajectories modeling and analysis. ACM Computing Surveys, 45(4):42:1-42:32.

Poushter, J. (2019). Pew research center: Smartphone ownership and internet usage continues to climb in emerging economies.

Raman, R., Raman, V., and Rao, S. (2002). Succinct indexable dictionaries with applications to encoding $k$ ary trees and multisets. In Proceedings of ACM-SIAM Symposium on Discrete Algorithms (SODA 2002), pages 233-242.

Schmid, F., Richter, K., and Laube, P. (2009). Semantic trajectory compression. In Proceedings of the Advances in Spatial and Temporal Databases International Symposium, SSTD 2009, volume 5644 of Lecture Notes in Computer Science, pages 411-416. Springer.
Song, R., Sun, W., Zheng, B., and Zheng, Y. (2014). PRESS: A novel framework of trajectory compression in road networks. Proceedings of the VLDB Endowment, 7(9):661-672.

Wyffels, J., De Brabanter, J., Crombez, P., Verhoeve, P., Nauwelaers, B., and De Strycker, L. (2014). Distributed, signal strength-based indoor localization algorithm for use in healthcare environments. IEEE Journal of Biomedical and Health Informatics, 18(6):1887-1893.

Yan, Z., Chakraborty, D., Parent, C., Spaccapietra, S., and Aberer, K. (2013). Semantic trajectories: Mobility data computation and annotation. ACM Transactions on Intelligent Systems and Technology, 4(3):49:149:38. 